\title{
Reliable Extraction of Cycling Induced Interface States Implementing Realistic P/E Stresses in Reference Cell: Comparison With Flash Memory Cell
}

\author{
Chang-Ki Baek, Bomsoo Kim, Younghwan Son, Wookhyun Kwon, Chan-Kwang Park, Young June Park, \\ Hong Shick Min, and Dae M. Kim
}

\begin{abstract}
The cycling induced interface states in floating-gate EEPROM cells are reliably extracted by implementing accurate program/erase stresses in the reference cell. The interface states measured directly from the memory cell via charge pumping are shown different from those obtained conventionally from the reference cell. The reasons for these different levels of extraction are elucidated and a new method is presented for accurate determination of interface trap density. The technique is based on introducing the equivalent gate voltage with offset voltage at the reference cell by which to simulate realistically the cycling stresses as occur in the flash memory cell itself.
\end{abstract}

Index Terms - Charge pumping, interface states, flash EEPROM cell.

\section{INTRODUCTION}

$\mathbf{T}$ HE degradation of the tunnel oxide by the program/erase $(\mathrm{P} / \mathrm{E})$ cycling is a major reliability concern in Flash EEPROM cells. The high field stresses entailed in Fowler-Nordheim (FN) tunneling and channel hot electron injection (CHEI) are responsible for generating interface and oxide traps [1]-[4]. The interface states decrease the cell subthreshold slope and transconductance, while increasing the off current [1], [4].

We present in this paper a reliable extraction of the cycling induced interface states $\left(N_{\text {it }}\right)$ by realizing accurate P/E stress conditions in the reference cell, where the control and floating gates are shorted. $N_{\mathrm{it}}$ is generally extracted from reference cell [1]-[4] but the FN and CHEI stress conditions used therein can be much different from those in memory cell. This is due to varying floating gate voltage $\left(V_{\mathrm{FG}}\right)$ resulting from charging and discharging in P/E. We show that $N_{\text {it }}$ 's extracted directly from the memory cell are different from those obtained conventionally from the reference cell. We propose a simple but reliable scheme of extracting $N_{\mathrm{it}}$ from the reference cell.

Manuscript received October 31, 2005. This work was supported by the NanoSystems Institute (NSI-NCRC) program sponsored by the Korea Science and Engineering Foundation (KOSEF) and in part by a Grant from Samsung Electronics, Inc. The review of this letter was arranged by Editor S. Chung.

C.-K. Baek, Y. J. Park, and H. S. Min are with the School of Electrical Engineering and Computer Science and Nano-systems Institute (NSI-NCRC), Seoul National University, Seoul 151-744, Korea.

B. Kim, Y. Son, and D. M. Kim are with the Korea Institute for Advanced Study, Seoul 151-744, Korea.

W. Kwon and C.-K. Park are with the Memory Division, Semiconductor Business, Samsung Electronics Company, Ltd., Kyungki-Do, 449-900, Korea.

Digital Object Identifier 10.1109/LED.2005.864178

\section{RESULTS AND DISCUSSIONS}

A stacked gate flash memory cell was used together with the reference cell. The operational and device parameters are specified at the outset: the aspect ratio of $W / L=0.38 / 0.28 \mu \mathrm{m}$; tunnel oxide thickness $\left(t_{\mathrm{OX}}\right)$ of $10 \mathrm{~nm}$; the equivalent interpoly ONO thickness $\left(t_{\mathrm{ONO}}\right)$ of $14.5 \mathrm{~nm}$, the area ratio of bottom-to-top dielectric $(w)$ of 1.78; the gate and drain coupling coefficient of $\alpha_{\mathrm{CG}}=0.55, \alpha_{D}=0.1$; the programming voltages at control gate, drain, source and bulk of $V_{\mathrm{CG}}=9 \mathrm{~V}$, $V_{D}=4.5 \mathrm{~V}, V_{S}=V_{B}=0 \mathrm{~V}$, for $t_{P}=1 \mu \mathrm{s}$; the erase voltages of $V_{\mathrm{CG}}=-16 \mathrm{~V}, V_{D}=V_{S}=V_{B}=0 \mathrm{~V}$, for $t_{E}=1 \mathrm{~ms}$. The reading is done by monitoring $I_{D}$ of $1 \mu \mathrm{A}$ at $V_{D}=0.8$ $\mathrm{V}$ in which $V_{\mathrm{FG}}$ is pinned at $1.7 \mathrm{~V}\left(\triangleq V_{\mathrm{TFG}}\right)$. The program and erase threshold voltages are set at the control gate by $V_{\mathrm{CGP}}=7.5 \mathrm{~V}, V_{\mathrm{CGE}}=2 \mathrm{~V}$, respectively.

We introduce the offset voltage $\left(V_{\text {offset }}\right)$ of $V_{\mathrm{FG}}$ in $\mathrm{P} / \mathrm{E}$. Fig. 1 shows the energy band of the floating gate cell at flat band (a), representing the onset of accumulation for erase and at channel inversion for programming and reading (b), with no excess charge, i.e., $Q_{\mathrm{FG}}=0$. With $V_{S}=V_{B}=0 \mathrm{~V}$ in $\mathrm{P} / \mathrm{E}$, the differential capacitance coupling equation is given by $d V_{\mathrm{FG}}=\alpha_{\mathrm{CG}} d V_{\mathrm{CG}}+\alpha_{D} d V_{D}+d Q_{\mathrm{FG}} / C_{T}$ and upon integration one can write

$$
V_{\mathrm{FG}}=\alpha_{\mathrm{CG}} V_{\mathrm{CG}}+\alpha_{D} V_{D}+Q_{\mathrm{FG}} / C_{T}+V_{\text {offset }} .
$$

Here, use was made of the fact that coupling capacitances and coefficients ( $\alpha$ 's) are nearly constant in channel inversion or accumulation. $V_{\text {offset }}$ is the constant of integration, which assumes different constant values in accumulation and inversion regions.

At flat band and for $Q_{\mathrm{FG}}=0, V_{D}=0 \mathrm{~V}, V_{\mathrm{FG}}=V_{\mathrm{CG}}=-1$ $\mathrm{V}$ [Fig. 1(a)]. Hence, from (1) $V_{\text {offset }}=-0.45 \mathrm{~V}$ for accumulation. Likewise, at the channel inversion and for $Q_{\mathrm{FG}}=0$ and $V_{D}=0.8 \mathrm{~V}, V_{\mathrm{FG}}=V_{\mathrm{TFG}}=1.7 \mathrm{~V}$. From $V_{\mathrm{TFG}}=V_{\mathrm{FB}}+2 \phi_{p}+$ $V_{\mathrm{OX}}$, and the surface potential $\phi_{p}=0.495 \mathrm{~V}, V_{\mathrm{OX}}=1.71 \mathrm{~V}$ and $V_{\mathrm{ONO}}=1.4 \mathrm{~V}$, since $\left(V_{\mathrm{ONO}} / t_{\mathrm{ONO}}\right) w=V_{\mathrm{OX}} / t_{\mathrm{OX}}$ from the flux theorem. Thus, $V_{\mathrm{CG}}=V_{\mathrm{ONO}}+V_{\mathrm{FG}}=3.09 \mathrm{~V}$ and inserting these in (1) $V_{\text {offset }} \approx 0 \mathrm{~V}$ for channel inversion. For $Q_{\mathrm{FG}} \neq 0, \Delta V_{\mathrm{CG}}=\Delta Q_{\mathrm{FG}} / C_{\mathrm{ONO}}$ is always added for compensation with $C_{\mathrm{ONO}}$ denoting the interpoly capacitance.

We next consider $Q_{\mathrm{FG}}$ in $\mathrm{P} / \mathrm{E}$ states. In reading the programmed cell, one can put in (1) $V_{\mathrm{FG}}=V_{\mathrm{TFG}}=1.7 \mathrm{~V}$, $V_{\text {offset }} \approx 0 \mathrm{~V}, V_{\mathrm{CG}}=V_{\mathrm{CGP}}=7.5 \mathrm{~V}, V_{D}=0.8 \mathrm{~V}$, obtaining $Q_{\mathrm{FG}} / C_{T}=-2.5$. Likewise, in reading the erased cell, again 


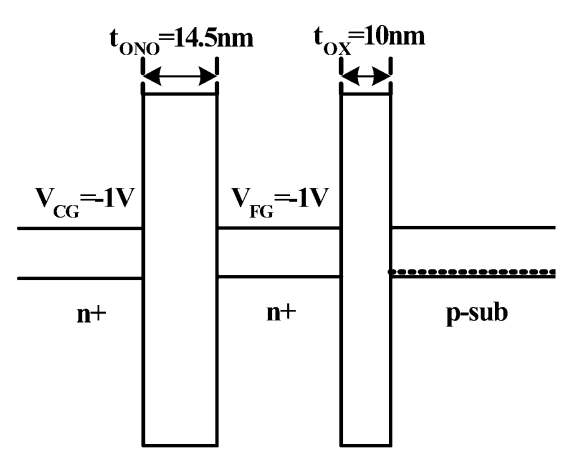

(a)

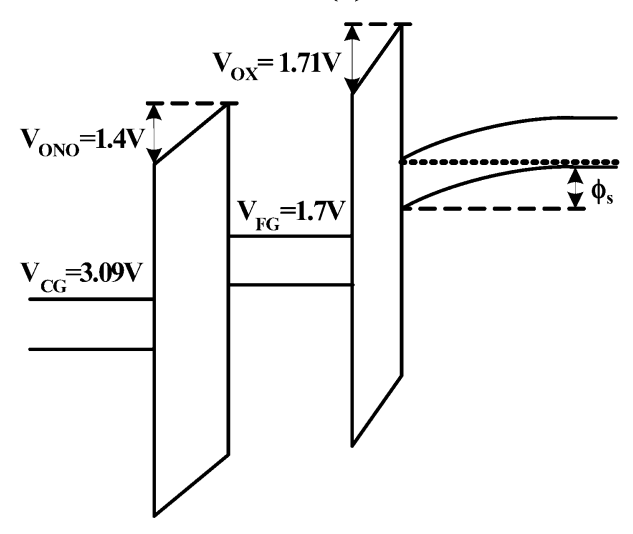

(b)

Fig. 1. Energy band diagrams of the floating gate memory cell.

$V_{\mathrm{FG}}=V_{\mathrm{TFG}}=1.7 \mathrm{~V}$, while $V_{\text {offset }} \approx 0 \mathrm{~V}, V_{\mathrm{CG}}=V_{C G E}=2$ $\mathrm{V}, V_{D}=0.8 \mathrm{~V}$ and therefore $Q_{\mathrm{FG}} / C_{T}=0.52$. Hence, the $Q_{\mathrm{FG}}$ swing renders $V_{\mathrm{FG}}$ vary from $-11.75 \mathrm{~V}$ to $-8.73 \mathrm{~V}$ during erase where $V_{\mathrm{CG}}=-16 \mathrm{~V}, V_{D}=0, V_{\text {offset }}=-0.45 \mathrm{~V}$. Similarly $V_{\mathrm{FG}}$ ranges from $5.92 \mathrm{~V}$ to $2.9 \mathrm{~V}$ during programming where $V_{\mathrm{CG}}=9 \mathrm{~V}, V_{D}=4.5 \mathrm{~V}, V_{\text {offset }} \approx 0$. This wide variation of $V_{\mathrm{FG}}$ renders it difficult to simulate $\mathrm{P} / \mathrm{E}$ stresses in the reference cell.

We present a new scheme for implementing such simulation and experimentally verify its validity. Fig. 2 shows P/E characteristics (a, b) and the schematics of $Q_{\mathrm{FG}}$ (c) and its drive gate current, $I_{G}(\mathrm{~d})$ in erase, for example. The trap generation is commensurate with the number of electrons traversing the tunnel oxide. Thus, one should find an equivalent gate voltage in reference cell by which to provide identical P/E fluency. Such a scheme is illustrated by choosing the erase as an example. Now $Q_{\mathrm{FG}}$ and $V_{T}$ are related by $\Delta Q_{\mathrm{FG}}=C_{\mathrm{ONO}} \Delta V_{T}$, so that $I_{G}\left(=d Q_{\mathrm{FG}} / d t\right)$ is given by $I_{G}=C_{\mathrm{ONO}} d V_{T} / d t$. We express the total change $Q_{\mathrm{TOT}}$ in erase by the given erase time by $Q_{\mathrm{TOT}}=I_{\mathrm{GEQ}} \cdot t_{E}$. The $I_{\mathrm{GEQ}}$ set in this manner is the gate current, evaluated at $Q_{\mathrm{EQ}}$, or equivalently at $\Delta V_{\mathrm{TEQ}}$ at $t_{\mathrm{EQ}}$ (see Fig. 2). In this case the local slopes at $Q_{\mathrm{EQ}}, V_{\mathrm{TEQ}}$ are identical to respective average slopes for erase [Fig. 2(b) and (c)].

If $t_{\mathrm{EQ}}$, hence $Q_{\mathrm{EQ}}, V_{\mathrm{TEQ}}$ are found, the total $V_{T}$-shift and $Q_{\mathrm{TOT}}$, i.e., the $Q_{\mathrm{FG}}$-swing in erase can be uniquely specified by a dc gate bias as follows. First, $V_{\mathrm{TEQ}}$ is found from equating the local slope with the average slope to be found from $V_{T}-t$ data, i.e., $V_{\mathrm{TEQ}}=3.1 \mathrm{~V}$ in the example discussed (see Fig. 2(b)).
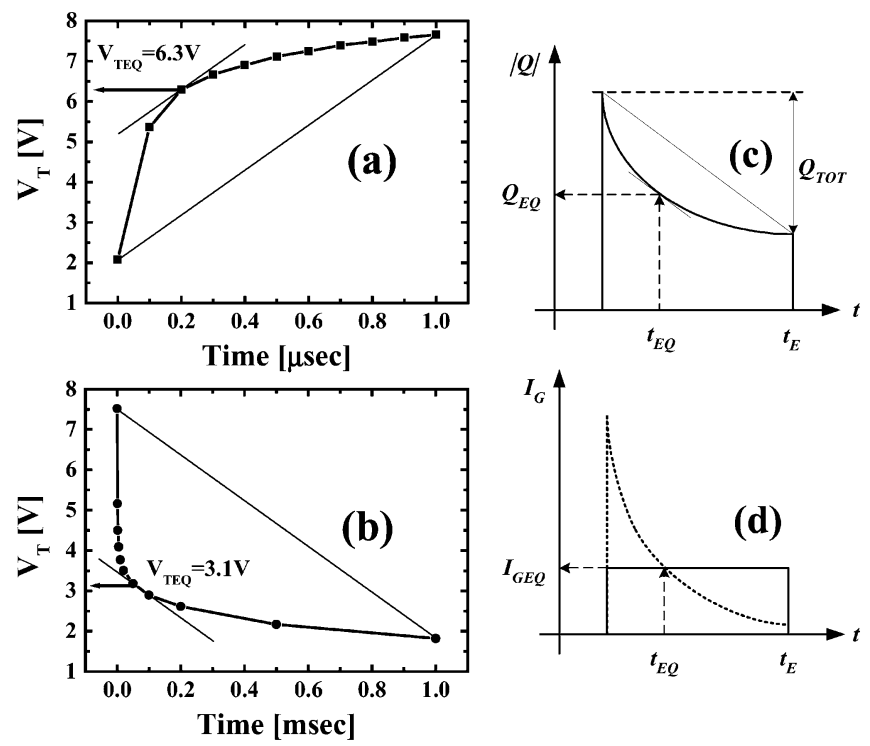

Fig. 2. Threshold voltage shift in (a) programming and (b) erase versus time. The schematics for (c) the excess charge and (d) the drive gate current in erase (or program).

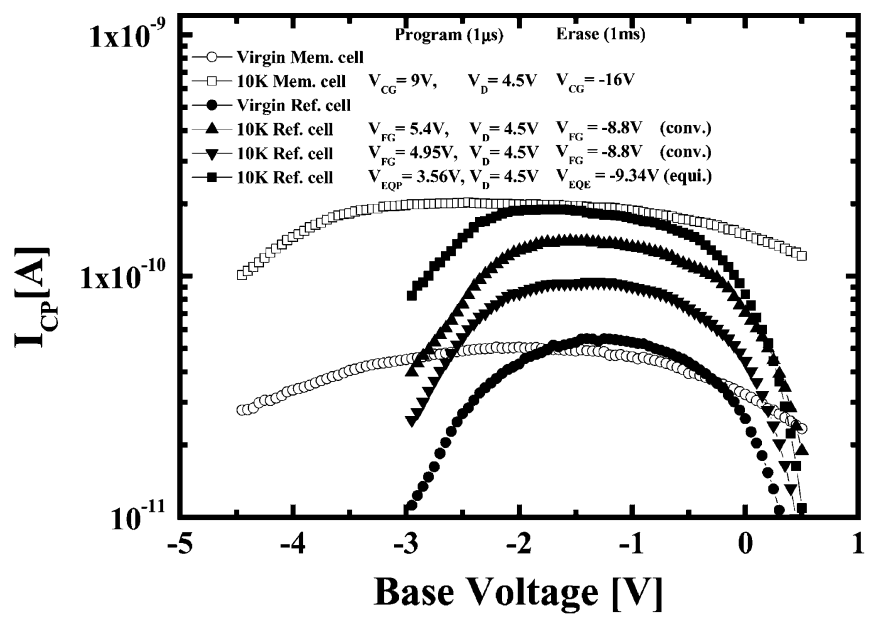

Fig. 3. Charge pumping current versus base voltage: virgin $(\mathrm{O})$ and $10-\mathrm{K}$ cycled memory cell $(\square)$; virgin reference cell $(\bullet) ; 10-\mathrm{K}$ cycled reference cells with conventional P/E stresses $(\boldsymbol{\Lambda}),(\boldsymbol{\nabla})$, and with equivalent P/E stress $(\boldsymbol{\square})$.

Next, by identifying $V_{\mathrm{CG}}=V_{\mathrm{TEQ}}$ in (1) and inserting reading biases, $V_{\mathrm{FG}}=V_{\mathrm{TFG}}=1.7 \mathrm{~V}, V_{D}=0.8 \mathrm{~V}, V_{\text {offset }} \approx 0 \mathrm{~V}$, one finds $Q_{\mathrm{EQ}} / C_{T}=-0.085$. By inserting this $Q_{\mathrm{EQ}} / C_{T}$ back into (1) with erase biases, viz. $V_{\mathrm{CG}}=-16 \mathrm{~V}, V_{D}=0 \mathrm{~V}$, $V_{\text {offset }}=-0.45 \mathrm{~V}$, one finds the equivalent erase gate voltage in the reference cell, viz. $V_{\mathrm{EQE}}=-9.34 \mathrm{~V}$. By repeating identical steps in program one can find $V_{\mathrm{EQP}}=3.56 \mathrm{~V}$.

Fig. 3 shows the charge pumping $(\mathrm{CP})$ current, $I_{\mathrm{CP}}$ [5], measured from memory and reference cells, using $\mathrm{CP}$ voltages of 6 $\mathrm{V}$ and $3.3 \mathrm{~V}$, respectively at $5 \mathrm{MHz}$ for rejection of the border trap effects [6] before and after $10 \mathrm{~K}$ cycles. Here, a small reverse bias of $V_{S}=V_{D}=50 \mathrm{mV}$ was applied. The maximum $I_{\mathrm{CP}}$ levels $\left(I_{\mathrm{CPMAX}}\right)$ from virgin memory and reference cells are shown identical, as it should. The only difference is the wider scan of $V_{\mathrm{CG}}$ for inducing similar $V_{\mathrm{FG}}$ swing in the reference cell. After $10 \mathrm{~K}$ cycling, $I_{\mathrm{CPMAX}}$ of the memory cell increases from $\approx 54 \mathrm{pA}$ to $\approx 200 \mathrm{pA}$ obviously due to stress induced $N_{\mathrm{it}}$. 

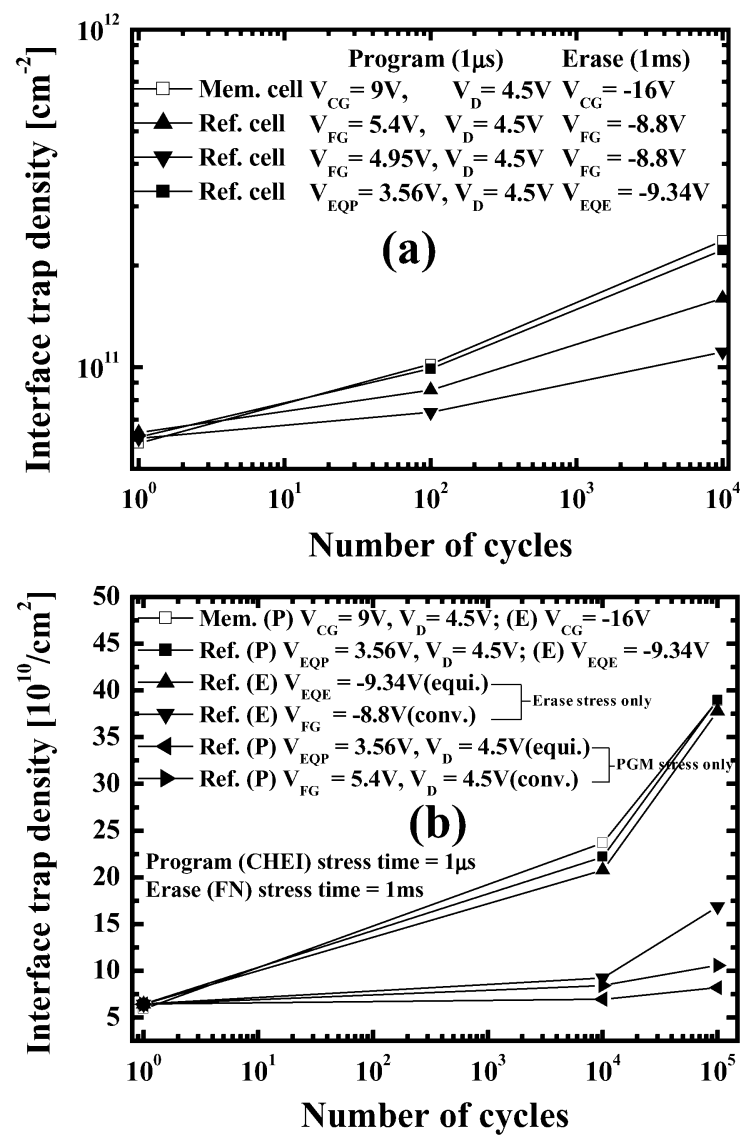

Fig. 4. Interface trap density versus P/E cycling: (a) memory cell ( $\square$ ); reference cells with conventional P/E stresses $(\boldsymbol{\Lambda}),(\boldsymbol{\nabla})$, and with equivalent $\mathrm{P} / \mathrm{E}$ stress $(\boldsymbol{\square})$. (b) Memory cell $(\square)$; reference cells with equivalent P/E stress $(\boldsymbol{\square})$, with equivalent erase (FN) stress only $(\mathbf{\Lambda})$ and conventional erase (FN) stress only $(\boldsymbol{\nabla})$, and with equivalent program (CHEI) stress only ( 4$)$ and conventional program (CHEI) stress only $(\bullet)$.

The same increase in $I_{\mathrm{CPMAX}}$ results from the reference cell which was stressed by the equivalent voltages, $V_{\mathrm{EQP}}=3.56 \mathrm{~V}$ and $V_{\mathrm{EQE}}=-9.34 \mathrm{~V}$ found in this work for P/E stress. This agreement in $I_{\mathrm{CPMAX}}$ proves the validity of the present stress scheme. Fig. 3 also shows $I_{\mathrm{CP}}$ 's from conventionally stressed reference cells, whose $I_{\text {CPMAX }}$ values are consistently lower than the true level. The conventional stress biases are found by using the coupling coefficients, viz. for programming by $V_{\mathrm{FG}}=$ $\alpha_{\mathrm{CG}} V_{\mathrm{CG}}+\alpha_{D} V_{D}=5.4 \mathrm{~V}$, or $V_{\mathrm{FG}}=\alpha_{\mathrm{CG}} V_{\mathrm{CG}}=4.95 \mathrm{~V}$, with $V_{D}=4.5 \mathrm{~V}$, while for erase by $V_{\mathrm{FG}}=\alpha_{\mathrm{CG}} V_{\mathrm{CG}}=-8.8 \mathrm{~V}$.
Fig. 4(a) plots $N_{\text {it }}$ versus P/E cycle calculated from $N_{\text {it }}=$ $I_{C P M A X} /$ qWLf [5] and in Fig. 4(b) the separate effects of program or erase stress on $N_{\mathrm{it}}$ in the reference cell are also presented. The present scheme reliably reproduces P/E stress induced $N_{\text {it }}$ up to $10^{5}$ cycles, in contrast with the conventional schemes yielding consistently lower $N_{\text {it }}$ values. Generally, the program and erase stress effects have been separately investigated in the reference cell [1], [2]. It is clear from Fig. 4(b) that $N_{\text {it }}$ is primarily generated by the erase stress and the reasons for the conventional schemes underestimating $N_{\text {it }}$ are clearly identified to be the erroneous choice of erase $V_{\mathrm{FG}}$. Finally, $N_{\mathrm{it}}$ due to cycling stress can be larger than the virgin level by an order of magnitude.

\section{CONCLUSION}

The equivalent floating-gate voltage $\left(V_{\mathrm{FG}}\right)$ considered at the reference cell by incorporating the offset voltages is shown to reproduce the actual $\mathrm{P} / \mathrm{E}$ stress, yielding essentially identical $N_{\text {it }}$ extraction as in the memory cell. Thus, the scheme enables useful utilization of the reference cell for fast examining interface states and also for examining separately the effect of program or erase stresses on $N_{\text {it }}$.

\section{REFERENCES}

[1] J.-D. Lee, J.-H. Choi, D. Park, and K. Kim, "Effects of interface trap generation and annihilation on the data retention characteristics of flash memory cells," IEEE Trans. Electron Devices, vol. 4, no. 3, pp. 110-117, Mar. 2004

[2] N.-K. Zous, Y.-J. Chen, C.-Y. Chin, W.-J. Tsai, T.-C. Lu, M.-S. Chen, W.-P. Lu, T. Wang, S. C. Pan, and C.-Y. Lu, "An endurance evaluation method for flash EEPROM," IEEE Trans. Electron Devices, vol. 51, no. 5, pp. 720-725, May 2004.

[3] S. S. Chung, C.-M. Yih, S.--M. Cheng, and M.--S. Liang, "A new technique for hot carrier reliability evaluations of flash memory cell after long-term program/erase cycles," IEEE Trans. Electron Devices, vol. 46, no. 9, pp. 1883-1889, Sep. 1999.

[4] S. Yamada, Y. Hiura, T. Yamane, K. Amemiya, Y. Ohshima, and K. Yoshikawa, "Degradation mechanism of flash EEPROM programming after program/erase cycles," in IEDM Tech. Dig, 1993, pp. 23-26.

[5] G. Groeseneken, H. E. Maes, N. Beltrán, and R. F. De Keersmaecker, "A reliable approach to charge-pumping measurements in MOS transistors," IEEE Trans. Electron Devices, vol. 31, no. 1, pp. 42-53, Jan. 1984.

[6] B. Djezzar, A. Smatti, and S. Oussalah, "A new oxide-trap based on charge-pumping (OTCP) extraction method for irradiated MOSFET devices: Part II (low frequencies)," IEEE Trans. Nucl. Sci., vol. 51, no. 4, pp. 1732-1736, Aug. 2004. 